\title{
SciencesPo
}

\section{ENTREPRENEURIAT ET CRÉATIVITÉ : DU DÉTOURNEMENT À LA CRÉATION DE VALEUR}

Jean-Luc Gaffard 


\section{SciencesPo}

EDITORIAL BOARD

Chair: Xavier Ragot (Sciences Po, OFCE)

Members: Jérôme Creel (Sciences Po, OFCE), Eric Heyer (Sciences Po, OFCE), Lionel Nesta (Université Nice Sophia Antipolis), Xavier Timbeau (Sciences Po, OFCE)

\section{CONTACT US}

OFCE

10 place de Catalogne | 75014 Paris | France

Tél. +33144185424

$\underline{w w w . o f c e . f r}$

WORKING PAPER CITATION

This Working Paper:

Jean-Luc Gaffard

Entrepreneuriat et créativité : du détournement à la création de valeur Sciences Po OFCE Working Paper, $\mathrm{n}^{\circ}$ 08/2020.

Downloaded from URL: www. ofce.sciences-po.fr/pdf/dtravail/WP2020-08.pdf DOI - ISSN 


\title{
ABOUT THE AUTHORS
}

Jean-Luc Gaffard, Institut Universitaire de France, OFCE Sciences Po, Université Côte d'Azur, CNRS, GREDEG, Skema Business School

Email Address: jeanluc.gaffard@sciencespo.fr

\section{RÉSUMÉ}

De la capacité de l'entrepreneur à concevoir une organisation apte à créer des canaux d'information suffisamment robustes dépend la nature des innovations effectuées, la façon dont sont exploitées les connaissances scientifiques et techniques, l'usage qui est fait de l'esprit créatif. C'est à cette aune qu'il convient de juger du comportement entrepreneurial, des rythmes imposés par la finance, de la façon dont sont allouées les ressources humaines. La réalité de la créativité réside dans le choix opéré entre recherche de rentes et innovations productives, entre une finance impatiente et une finance patiente, entre flexibilité et rigidité sur les marchés de travail. C'est un choix d'ordre organisationnel et institutionnel.

\begin{abstract}
The nature of the innovations carried out, the way in which scientific and technical knowledge are exploited, the use made of the creative spirit, depend on the entrepreneur's ability to design an organisation capable of creating sufficiently robust information channels. This is the yardstick by which to judge entrepreneurial behaviour, the pace imposed by finance, the way human resources are allocated. The reality of creativity lies in the choice between rent-seeking and productive innovation, between impatient and patient finance, between flexibility and rigidity in labour markets. This is an organizational and institutional choice.
\end{abstract}

\section{KEY WORDS}

Créativité, engagement, entrepreneuriat, innovation

JEL

D21, D23, D47, J53, L26 



\title{
Entrepreneuriat et créativité : du détournement à la création de valeur
}

\author{
Jean-Luc Gaffard \\ Institut Universitaire de France, OFCE Sciences Po, Université Côte d'Azur, CNRS, \\ GREDEG, Skema Business School
}

\section{Résumé :}

De la capacité de l'entrepreneur à concevoir une organisation apte à créer des canaux d'information suffisamment robustes dépend la nature des innovations effectuées, la façon dont sont exploitées les connaissances scientifiques et techniques, l'usage qui est fait de l'esprit créatif. C'est à cette aune qu'il convient de juger du comportement entrepreneurial, des rythmes imposés par la finance, de la façon dont sont allouées les ressources humaines. La réalité de la créativité réside dans le choix opéré entre recherche de rentes et innovations productives, entre une finance impatiente et une finance patiente, entre flexibilité et rigidité sur les marchés de travail. C'est un choix d'ordre organisationnel et institutionnel.

Mots clés : créativité, engagement, entrepreneuriat, innovation

Codes JEL : D 21, D 23, D 47, J 53, L 26

\begin{abstract}
:
The nature of the innovations carried out, the way in which scientific and technical knowledge are exploited, the use made of the creative spirit, depend on the entrepreneur's ability to design an organisation capable of creating sufficiently robust information channels. This is the yardstick by which to judge entrepreneurial behaviour, the pace imposed by finance, the way human resources are allocated. The reality of creativity lies in the choice between rent-seeking and productive innovation, between impatient and patient finance, between flexibility and rigidity in labour markets. This is an organizational and institutional choice.
\end{abstract}

Key words: commitment, creativity, entrepreneurship, innovation 


\section{Introduction}

Le concept de créativité est peu usité des économistes qui préfèrent, le plus souvent, se référer au concept d'innovation. Phelps (2013) fait exception dans sa tentative de comprendre les ressorts de ce qu'il dénomme l'économie moderne. Dans sa perspective, les nouvelles idées sont le moteur de l'histoire économique. La diversité des individus et l'interaction sociale permettent à ces idées de se combiner et de se multiplier. La sélection qui en résulte est la source d'une instabilité aussi inévitable que nécessaire. Concrètement, « une économie moderne laisse libre cours à la créativité et à la vision, mais parvient à les atteler dans une certaine mesure à l'expertise des entrepreneurs, au jugement des financiers et au discernement de l'utilisateur final » (Phelps 2013 p. 54). En contrepoint, Phelps dénonce les entraves à la créativité et à la création de richesses nées d'un nouveau corporatisme perpétuant et complétant le corporatisme de l'entre-deux guerres, et renvoyant à une série d'objectifs que sont « l'intervention de l'État plutôt que le désordre, la solidarité plutôt que l'individualisme, la responsabilité sociale plutôt que les comportements antisociaux (...), la codétermination plutôt que le contrôle exclusif des actionnaires et une prise en compte des parties prenantes plutôt qu'une carte blanche aux entreprises soucieuses d'optimiser les profits à répartir entre actionnaires et travailleurs » (Phelps 2013/2017 p. 203).

Cette position, fortement connotée idéologiquement, a le mérite de poser la question de la créativité en termes de choix de société. Implicitement, éloge est fait de ce qu'il est convenu d'appeler le néo-libéralisme en dépit des déboires et désordres suscités par ce dernier, mais finalement attribués par Phelps à la survivance de structures institutionnelles caractéristiques du libéralisme social ici assimilé à un nouveau corporatisme. La société et l'économie vantées sont celles des petits propriétaires dont les idées nouvelles viennent remettre en cause systématiquement les positions acquises et nourrir la création de richesses. Le monde ainsi décrit est largement idéalisé, ne se heurtant à aucun obstacle autre que de mauvaises institutions sans lesquelles un ordre spontané prévaudrait conformément à la thèse défendue par Hayek (1948). De là, la défiance à l'égard de la grande entreprise managériale et de la banque d'investissement ou des fonds de placement dont le comportement irait et peut effectivement aller à 
l'encontre des innovations et des investissements d'avenir systématiquement repoussés (Phelps ibid. p. 288-89).

Cette façon de voir les choses ne fait guère cas du rôle de l'organisation (de l'entreprise) dans une économie de marché. Or, dans situation d'incertitude radicale qui est celle dans laquelle se place Phelps se référant sur ce point à Keynes, alors que le système de prix est défaillant à assurer une coordination efficace y compris en termes de sélection et de destruction créatrice, le culte du marché pas plus que celui de l'État ne sauraient prévaloir. L'action collective apparaît nécessaire pour autant qu'elle étend le domaine de la rationalité individuelle, qu'elle constitue le moyen par lequel les individus parviennent à pleinement réaliser leurs valeurs individuelles (Arrow 1974 p. 16). Dès lors, « loin d'obéir à de pures motivations économiques, des relations économiques continues finissent souvent par être subordonnées à un environnement social qui porte à être fortement confiant dans le futur et à s'abstenir de comportements opportunistes ». (Granovetter, 1985 p. 490)

L'entrepreneur est la figure centrale de cet environnement. Cet entrepreneur est, certes, celui décrit par Schumpeter (1934) qui en fait l'agent de l'innovation entendue comme une rupture du flux circulaire (i.e. de l'équilibre existant), celui qui saisit une opportunité d'investir et de réaliser un profit. Mais il peut aussi exercer son talent créatif sans avoir de réelle contribution à l'activité productive et, même, dans certains cas, en ayant un rôle destructeur ainsi que le souligne Baumol (2002). Aussi la création de richesses dépendelle de l'allocation des talents d'entrepreneur entre des activités productives et des activités improductives, entre la création et le détournement de valeur. L'usage des ressources humaines et financières, que commande l'esprit créatif, dépend du contexte organisationnel et institutionnel dans lequel cette ressource est déployée.

Cette approche du problème de la créativité est loin d'être étrangère à la manière dont Smith (1776) s'enquiert de la question de la richesse des nations quand il reconnaît dans la division du travail la clé de la croissance économique et fait dépendre cette dernière, outre de l'extension des marchés, de l'offre de capital, et par suite de l'usage des fonds partagés entre emplois productifs et improductifs, l'intervention du gouvernement devant être spécialement restreinte quand elle s'exerce au bénéfice d'intérêts constitués qui pervertissent la création de richesse. 
Dans un contexte d'incertitude radicale, si l'on se rapporte à l'analyse de Shackle (1949, 1955) faisant référence au degré de surprise davantage qu'au degré de croyance, «bien que la survenue de tout événement pour lequel il existe un degré de croyance ex ante entraîne un degré de surprise (d'autant plus grand que le degré de croyance est petit), pour un événement vraiment nouveau, il existe une surprise ex post mais aucune croyance ex ante en cet événement » (Georgescu-Roegen 1971 p. 123). À défaut d'une telle croyance, l'événement se construit en chemin en réponse à l'environnement institutionnel et au degré de confiance qu'il autorise. « Le postulat de l'homo economicus maximisant son utilité en étant isolé de ses semblables ne peut pas avoir de sens dans un monde dans lequel nous ne savons pas ce que demain nous apportera. En même temps, l'incertitude explique l'importance que la société a toujours accordé aux institutions qui ont établi la confiance et ancré les anticipations. Il n'est pas besoin de se replier sur des explications neurologiques de l'existence de la société» (Skidelski 2009 p. 92). Dit autrement, « les gens ne sont pas en mesure d'agir suivant la théorie conventionnelle du choix rationnel s'ils ne peuvent pas attacher des probabilités numériques à toutes les conséquences possibles de leurs décisions (...) Dans ces circonstances, ils ont tendance à s'en sortir en recourant à la coutume et à la convention. Ils conçoivent aussi des institutions pour se protéger d'avoir à se reposer sur des prévisions nécessairement peu fiables » (Howitt 2008 p. 159).

La structure d'information requise par l'individu concerne, certes, l'état de la connaissance existante, mais aussi la possibilité de disposer dans le futur d'une information fiable. C'est pourquoi, «la valeur de la prise de décision hors marché, la désirabilité de créer des organisations d'ampleur plus limitée que ne l'est le marché global, est partiellement déterminée par les caractéristiques du réseau de flux d'information » (Arrow 1974 p. 37). De fait, il apparaît que les coûts d'acquisition de l'information sont un coût en capital et «représentent typiquement un investissement irréversible » (ibid. p. 39). En outre, l'acquisition d'information repose sur une approche commune aux acteurs du changement, le partage d'un même code et par suite la constitution de coalitions au cœur des formes requises d'organisation. «Si, comme l'a suggéré un jour Adam Smith, les membres d'un même commerce trouvent qu'il est facile de communiquer entre eux vraisemblablement en raison de leurs expériences communes, il se pourrait bien que l'échange d'informations menant à un accord collusif entre les producteurs d'un même produit soit beaucoup moins coûteux que celui nécessaire pour 
parvenir à une coalition de blocage. Par suite, l'accord collusif peut en fait être stable » (ibid. p. 42).

Dès lors, de la capacité de l'entrepreneur à établir une organisation propre à créer des canaux d'information suffisamment robustes dépend la nature des innovations effectuées, la façon dont sont exploitées les connaissances scientifiques et techniques, l'usage qui est fait de l'esprit créatif. C'est à cette aune qu'il convient de juger du comportement entrepreneurial (section 2), des rythmes imposés par la finance (section 3) et de la façon dont sont allouées les ressources humaines (section 4), en faisant le partage entre ce qui relève de la création et du détournement de valeur. Alors que le discours néo-libéral décrit la création de valeur comme le propre de l'individu débarrassé de toute contrainte, il apparaît qu'elle procède d'institutions de marché que l'on ne saurait réduire à une concurrence dite libre et non faussée.

\section{L'entrepreneur à la croisée des chemins}

L'activité entrepreneuriale, dont il faut convenir qu'elle est largement absente de la théorie économique dominante, peut être créative et d'une certaine manière innovante sans pour autant avoir une contribution significative à la croissance du produit, étant essentiellement, tournée vers la captation d'une rente (Baumol 2002 p. 59-62). Cette créativité consiste à saisir une opportunité et à la concrétiser rapidement. De quelque manière, l'activité de l'entrepreneur de ce que l'on appelé la nouvelle économie, ramené à l'individu, relève de cette catégorie. La création de richesses et l'obtention de gains de productivité demeurent, pourtant, l'apanage d'un entrepreneuriat essentiellement conçu comme une coalition politique entre différents acteurs - managers, salariés, détenteurs de capitaux, clients ou fournissers - porteuse de la dimension sociale de la créativité et de l'innovation (March 1962).

\section{Le nouvel entrepreneur ou la créativité immédiate}

Un nouveau modèle d'affaires semble prévaloir dans les esprits comme dans la pratique des affaires avec l'irruption des nouvelles technologies, qui n'est pas sans expliquer l'engouement pour l'idée de créativité. Il concerne les relations entre entreprises, la définition de leurs frontières, leur rapport à la finance et les conditions de gestion de l'emploi avec en filigrane l'apologie de la start-up censée être le véritable moteur de l'innovation. 
Suivant les termes de ce modèle, la spécialisation verticale des entreprises et le développement de systèmes technologiques ouverts (non appropriés) offrent des opportunités de création de nouvelles entreprises (y compris au terme de restructurations par fusions et acquisitions) qui s'emparent de nouvelles niches. Ces entreprises bénéficient du développement des sociétés de capital-risque, de l'ingéniosité de la finance et de son activisme via les marchés financiers. Elles cherchent à disposer de travailleurs qui ont une expérience de l'industrie en ayant déjà occupé des emplois dans cette industrie plutôt qu'une expérience accumulée dans la même entreprise. Le marché du travail est plus fluide, le taux de rotation des salariés est plus élevé avec, possiblement, à la clé des hausses de salaires des plus qualifiés mais aussi un remplacement plus systématique des salariés âgés par de plus jeunes. La valeur établie par les actionnaires (shareholder value) devient le critère de gestion dominant en tant qu'il justifie l'entrée et le maintien sur des marchés financiers réputés efficients qui sanctionnent le succès des choix technologiques et organisationnels (Lazonick 2009).

L'adoption de conceptions modulaires, forme moderne de la division du travail, que rend possible la flexibilité renforcée des systèmes de production, serait, ainsi, à l'origine d'une accélération du rythme des innovations en même temps que de l'augmentation de la capitalisation boursière des entreprises. L'explication proposée est simple dans son énoncé. L'architecture modulaire conduirait à une décomposition largement accrue des opérations productives devenues indépendantes les unes des autres. De nouveaux champs seraient ainsi ouverts aux investissements et à la concurrence, favorisant l'initiative individulle et, par suite, l'innovation et la croissance.

Une telle démultiplication des opportunités, quoique très forte, n'est pas censée menacer la stabilité du système dans son ensemble, la conviction étant qu'il y a beaucoup de nouveaux, beaucoup de perdants, et seulement un petit nombre de gagnants, mais que l'essentiel du risque disparait au niveau agrégé. La somme des valeurs initialement dispersées entre les différents expérimentateurs irait au gagnant (Baldwin et Clark 2001 p. 11).

Si tel était le cas, les investisseurs n'auraient pas à réviser leurs croyances sur la distribution de probabilités qui sous-tend les expérimentations. La structure de marché qui s'imposerait au terme du processus de sélection serait celle qui était rationnellement anticipée. Elle est essentiellement concurrentielle, non pas en raison du nombre de 
compétiteurs présents, mais du fait de la libre entrée et sortie du marché, de l'absence de coûts irrécouvrables. L'entrepreneur individuel joue, gagne ou perd sous l'effet de la sanction imposée par le marché. Parfois magnifié, il est, en réalité, le jouet des marchés qui exercent pleinement la fonction de coordination en sélectionnant toujours les meilleurs.

L'incertitude inhérente à l'innovation est, en quelque sorte, gommée du fait de la redistribution supposée des actifs au bénéfice du ou des gagnants. Si coût irrécouvrable il y a, il n'exerce aucune influence sur la décision d'un entrepreneur qui réalise une innovation unique et non, de manière routinière, une suite d'innovations (Baumol 2002 p. 38-39). Si les revenus d'une entreprise innovatrice s'avèrent insuffisants pour rembourser les dettes encourues du fait des coûts engagés dans le passé, cela affectera ses propriétaires qui font faillite. Mais cela n'affectera pas la société dans son ensemble, pas même l'industrie, dans la mesure où la faillite de cette entreprise se conclut par le transfert de ses actifs entre d'autres mains. La fluidité des marchés et l'instantanéité des adaptations sont les clés de l'évolution ainsi conçue.

Cet entrepreneur, s'il bénéficie, concrètement, d'un faible degré d'appropriation des protocoles de base, pourra s'engager dans des activités à faible coût d'entrée et immédiatement rentables dont il pourra se dégager non moins rapidement. Dans ce cas de figure, les gains de productivité sont, le plus souvent, faibles, la plupart des emplois créés peu ou pas qualifiés, mais la rentabilité possiblement élevée.

Ce monde est celui vanté par Phelps (2013) fait de créativité, de mobilité, d'instabilité mais aussi de continuité et de création de valeur sans destruction de resources. C'est un monde largement virtuel, hors du temps, quand on sait que les 'success stories' deviennent de grands groupes, que l'introduction en bourse change la nature des innovations qui deviennent incrémentales (mais très coûteuses), que les gains captés par les vainqueurs peuvent se transformer en rentes, étant utilisés à des achats de titres ou de biens de luxe plutôt qu'à réaliser des investissements productifs. Est-ce pour autant que la capacité de création de valeur disparaît? Non si l'on considère le rôle joué par l'organisation dans la création de connaissances.

\section{L'entrepreneur maître du temps ou la créativité sociale}

L'entrepreneur innovateur n'est pas seulement celui qui saisit une opportunité et fait preuve de créativité. Il doit avoir la capacité de prévoir, de planifier et de mener à son terme 
une activité productive dont la mise en œuvre prend du temps dans un contexte où les coûts surviennent avant les revenus qui leur correspondent. Sans la maîtrise du temps, il n'investira pas ou du moins il n'investira pas à long terme et pourrait se contenter de la captation d'une rente en jouant immédiatement de son pouvoir de marché.

La capacité entrepreneuriale, loin de relever de la seule intuition de l'inventeur est, dès lors, une capacité d'organisation. L'organisation aide à créer les connaissances nécessaires, en fait à mieux maîtriser un environnement technologique et de marché en mutation permanente (Marshall 1920, Loasby 1991). Elle repose sur l'existence d'un pool de ressources dont la gestion n'est pas guidée par la seule optimisation de leur usage immédiat (Penrose 1959). Elle s'inscrit dans les relations nouées entre les différentes parties prenantes dans l'entreprise (managers, actionnaires, banquiers, salariés, clients et fournisseurs), des relations aussi bien internes qu'externes, lesquelles témoignent de l'existence d'une base de connaissances constituant un actif spécifique de l'entreprise en question. Contrairement à ce qui est habituellement convenu, l'entreprise n'est pas cette unité de base rivée à un objectif de maximisation du profit impliquant d'en réserver la direction aux actionnaires, mais une coalition d'intérêts en même temps que le lieu de résolution des conflits entre ces différents intérêts. L'entreprise a pu être qualifiée de coalition politique entre une large gamme de participants potentiels. Différentes coalitions sont possibles qui correspondent à des intérêts différents suivant les groupes de participants mais compatibles entre eux. Elles ont chacune une valeur qui est celle de l'entreprise en relation avec son environnement (March, 1962).

Quand une entreprise planifie d'introduire un nouveau produit, d'investir dans une capacité additionnelle ou d'entrer sur un nouveau marché, elle doit pouvoir faire des anticipations fiables à propos de ce qu'il va advenir de son environnement de marché. Cette exigence informationnelle peut expliquer que l'entreprise applique des routines et qu'elle ne s'aventure pas dans des activités pour lesquelles elle ne disposerait pas préalablement des compétences requises. Mais elle explique surtout le type de régime de concurrence ou d'organisation des marchés qui lui permet de pouvoir s'engager de manière irréversible et de faire face aux contraintes d'un choix innovateurs. Les formes d'organisation qui structurent les marchés concurrentiels sont les véritables processeurs d'information et de connaissance (Metcalfe 2001). 
Les entreprises installées, qui réalisent des innovations incrémentales, savent qu'elles sont plusieurs à envisager le même type d'investissement et entendent trouver le moyen de se coordonner afin d'éviter de trop investir en relation avec la demande qui leur sera finalement adressée. Elles ne prendront rationnellement des décisions d'investir que si elles disposent d'une information minimale sur le comportement à venir des autres acteurs du processus d'innovation, en fait si elles sont capables de construire avec eux le nouveau marché au moyen de connexions appropriées qui ne sont rien d'autres que ce que l’on dénomme habituellement des imperfections de marché (Richardson 1960). L'objectif, pour chaque entreprise, est de calibrer et par suite de sécuriser ses investissements de telle sorte que la production future rencontre une demande équivalente et que l'exigence de rentabilité soit satisfaite. Ce calibrage implique que les plans des différentes entreprises concurrentes ou complémentaires s'avèrent compatibles entre eux. La compatibilité recherchée ne procède pas du système de prix. Elle est le fruit de stratégies de marché qui ont une double dimension concurrentielle et coopérative (Richardson 1960, Metcalfe 2001).

L'incitation à investir est subordonnée à l'existence de ces connexions, c'est-à-dire à une forme spécifique de coordination inter-temporelle qui accroît la fiabilité des anticipations à long terme en faisant en sorte qu'elles soient partagées entre les différentes parties prenantes du processus de marché. La disponibilité ou, plus exactement, la création de l'information de marché pertinente dépend de l'existence de restrictions ou de contraintes naturelles ou contractuelles sur l'investissement qui réduisent la liberté d'action de chaque entreprise en même temps qu'elles rendent crédible pour elle d'innover.

Les arrangements les plus fréquents sont ceux qui donnent lieu à la constitution de réseaux entre entreprises concurrentes, à commencer par les accords de coopération en R\&D et les autres formes de consortia technologiques (Baumol 2002 p. 93 et sq.). Autant de moyens, non seulement de diminuer les coûts, mais aussi et surtout de partager une information sur la configuration future des marchés rendant crédible pour chacun de s'engager. Le comportement des entreprises parties prenantes de ces arrangements va dépendre de leur horizon temporel. Il est à parier que plus cet horizon est long, moins les comportements anti-concurrentiels sont vraisemblables.

Des arrangements organisationnels sont, également, nécessaires le long de la chaîne de valeur, entre l'amont et l'aval. Ces arrangements, qualifiés de restrictions verticales, ont 
pour but de coordonner des investissements complémentaires afin de les rendre mutuellement crédibles. Ainsi les pratiques d'exclusivité dans la distribution de produits ont pour objectif de sécuriser les investissements des distributeurs en même temps que ceux des producteurs sans quoi il n'y aurait pas d'incitations, pour les uns comme pour les autres, à les effectuer. Des restrictions, qui peuvent être perçues comme relevant d'une action anti-concurrentielle, sont en réalité ce qui permet à l'entreprise en amont comme à l'entreprise en aval de s'engager dans des dépenses d'investissement dont la réalisation prend du temps. Elles préservent ainsi la concurrence par l'innovation en réduisant les risques encourus par chacune des entreprises partenaires.

Ces connexions de marché, souvent qualifiées d'imperfections de marché, constituent, certes, des pratiques monopolistes, mais elles jouent un rôle essentiel dans la conduite des processus d'innovation (Schumpeter 1941). La qualification du revenu obtenu dépend de l'usage qui en est fait. S'il est investi dans des activités productives dont le déroulement prend du temps et s'il permet d'accroître l'offre future de biens ou de services, ce n'est pas, au sens propre une rente en dépit de l'appellation qui leui est donnée dans la théorie économique standard. Ce n'est que s'il est détourné de ces activités qu'il devient une rente en étant, par exemple consacré à des rachats d'actions au détriment de l'investissement productif. En d'autres termes, la création de valeur est subordonnée à l'usage des ressources disponibles, ce qui pose inévitablement la question du rôle de la finance.

\section{L'ambivalence de la finance}

L'innovation est indissociable du crédit (Schumpeter 1934). Le mode de financement la nature de l'intermédiaire financier et du contrat de financement - conditionne les choix d'affectation de l'épargne et concourt au partage entre une activité d'innovation et la recherche de rentes. De même qu'il existe deux types d'entrepreneurs, il existe deux types d'intermédiaires financiers suivant le rapport qu'ils entretiennent avec le temps. Les uns, actionnaires ou banquiers, s'engagent dans la durée avec les entreprises qu'ils financent, les autres spéculent à court terme. La nature de l'intermédiation financière conditionne celle de l'entrepreneuriat, autrement dit l'exercice de la créativité.

\section{L'idéologie du contrôle ou le pouvoir exclusif des actionnaires}

Le management par les marchés financiers constitue une rupture avec le management 'traditionnel' de la grande entreprise née au tournant du 19 ème et 20 ème siècle. Cette rupture 
est associée à l'évolution récente de l'architecture de l'organisation industrielle, notamment dans les activités liées aux technologies de l'information et de la communication, mais aussi dans le domaine des biens faisant l'objet d'assemblages complexes comme l'automobile ou les appareils électroniques. La grande entreprise intégrée verticalement cède devant l'émergence et le développement de larges grappes modulaires d'entreprises et de marchés. Les modules sont des nœuds de réseaux liant de multiples clients et fournisseurs, révélant de nombreux liens de complémentarité et dont le périmètre évolue avec le temps et les opportunités. Ce que l'on dénomme, traditionnellement, la division du travail se poursuit. Elle opère moins au sein d'une même entreprise qu'entre de nombreuses entreprises aux activités complémentaires liées par des relations de marché.

Les marchés financiers sont, alors, présentés comme le mode de financement le plus apte à valoriser ces projets inscrits dans une architecture modulaire. Ils auraient la capacité de prendre en considération les valeurs d'option associées à chaque projet particulier. Ce rôle attribué aux marchés financiers tient à la dispersion des modules et à celle de l'information les concernant qui rendent peu opérantes les stratégies bancaires classiques. Le management par la valeur des actions encourage les managers à mettre en place ces architectures modulaires et à jouer de la création d'options. Il encourage les managers à se projeter dans le futur sans que l'on ait à craindre que leurs erreurs puissent conduire, globalement, à un gaspillage de ressources productives.

La flexibilité des mécanismes de financement va ici de pair avec la flexibilité ou la malléabilité de l'organisation industrielle, la démultiplication des modules au bénéfice de la croissance de l'ensemble de l'économie. Le mode de financement en question est supposé neutre dans la mesure où il permet seulement de faire valoir les avantages réels nés des nouvelles opportunités technologiques.

Les avantages ainsi prêtés aux marchés financiers conduisent à considérer l'entreprise comme une collection de droits de propriété assimilable à un ensemble d'instruments financiers comprenant, d'une part, des droits et des créances et, d'autre part, des actifs en place et des options de croissance. Le principe est simple. Tout actif réel est susceptible d'être acheté ou vendu et cette transaction aura un effet sur la valeur de l'action. Par analogie avec les actifs financiers, il est possible de vendre ou d'acheter à tout moment des actifs comparables ou de réinvestir les fonds sur d'autres opportunités. L'option fait 
donc partie du choix et constitue un facteur important de flexibilité. Le risque global d'un actif non financier s'analyse de la même façon que celui d'un actif financier. La valeur créée résulte de la variation nette de l'ensemble des actifs et des passifs évalués à leur valeur de marché. On tend alors vers une situation où le bilan utile est un bilan en valeur de marché censée refléter la vraie valeur de l'entreprise au sens d'une valeur de long terme si tant est que la distinction entre court et long terme ait ici un sens. L'entreprise voit ses contours et la nature de son activité déterminés par un agent extérieur que sont les marchés financiers. Elle n'existe pas par elle-même.

Le principe de gouvernance fait une place exclusive au contrôle que doivent exercer les actionnaires sur les managers de l'entreprise. L'orientation des choix d'investissement est commandée par le souci de maximiser la valeur des actions. Or la concentration de la propriété des actions entre les mains de fonds de placement activistes conduit à faire dépendre celle-ci des résultats trimestriels de l'entreprise dont l'horizon temporel se trouve ainsi singulièrement réduit. Les investissements réalisés ont, alors, un temps de gestation de plus en plus court quand bien même les fonds disponibles ne sont pas affectés à des rachats d'action au détriment de l'investissement productif. La créativité, y compris financière, s'exerce, sinon au détriment de la création de valeur, du moins à celui d'investissements à long terme frappés d'incertitude radicale mais aussi de gains de productivité significativement élevés (Mazzucato 2013).

\section{L'engagement financier ou la socialisation du choix innovateur}

La réalité financière peut être toute différente. De fait, «la condition sociale qui peut transformer la finance en innovation est l'engagement financier : un ensemble de relations qui assure l'allocation de fonds pour soutenir le processus d'innovation cumulatif jusqu'à ce qu'il génère des rendements financiers » (Lazonick 2007 p. 999). En d'autres termes, la distinction pertinente est celle entre la finance patiente et la finance impatiente, que l'innovation soit radicale ou progressive. D'autant, d'ailleurs, que les innovations majeures n'existent, le plus souvent, qu'en raison, de financements publics massifs associé à la mise en œuvre de grands programmes à longue échéance (Mazzucato 2013, 2014). La question pertinente concerne la durée de la période de gestation de l'investissement, que détermine la nature du financement, dans la mesure où plus cette durée est longue, plus le détour de production est grand, plus les gains de productivité sont élevés. «Ce que l'on appelle souvent le capital 'patient' permet aux capacités qui 
découlent de l'apprentissage collectif de s'accumuler dans le temps, malgré l'incertitude inhérente au processus d'innovation » (Lazonick 2007 p. 999).

L'engagement financier (une finance patiente) n'est vulnérable ni au surgissement de temps difficiles inhérents à tout processus d'innovation, ni aux tentations de changement brusque d'orientation. Il consiste dans le montant des capitaux engagés et la durée de leur engagement. Un montant important engagé qui peut être retiré instantanément est de peu de valeur, de même que peu de capital engagé pour une longue période (Mayer 2013 p. 145). Dans ces conditions, «le contrôle stratégique des revenus internes est une forme essentielle d'engagement financier, mais ce 'capital interne' doit souvent être complété par des sources de financement externes telles que des émissions d'actions, d'obligations ou de dettes bancaires qui, à différents moments et en différents lieux, peuvent être plus ou moins engagées pour soutenir le processus d'innovation » (ibid.) Ce qui est, finalement, en jeu, ce sont aussi bien la structure de l'actionnariat (le poids des fonds communs de placement) que celle du système bancaire (le poids des banques d'investissement), car elles sont déterminantes du degré d'engagement des détenteurs de capital.

Le propre de l'engagement financier est qu'il soutient l'engagement des autres parties prenantes dans l'entreprise que sont les fournisseurs, les clients et les salariés. Les uns et les autres effectueront les investissements nécessaires en ayant cette garantie que leur offrent les détenteurs de capitaux. Ce qui peut paraître paradoxal, mais ne l'est qu'en apparence, est que l'engagement réduit la liquidité au sens étroit de négociabilité des actifs, restreint la gamme des options qui pourraient être choisies en cours de route par l'un ou l'autre acteur, limite les choix ultérieurs autrement dit la créativité. En fait, sans ces limites ou restrictions il n'y aurait pas d'engagement, pas de possibilité de répondre à l'irréversibilité et à l'incertitude qui caractérisent les investissements innovants. Elles garantissent la capacité de l'entreprise de prévoir et de planifier.

Le financement de marché permet, dans son principe, un engagement long, puisque les émissions d'actions souscrites sont constitutives d'un capital permanent. Certes, l'existence d'un actionnariat dispersé et la constitution de la grande entreprise se sont traduits par la séparation entre la propriété et le contrôle donnant lieu à de possibles inefficiences. Le contrôle ayant été transféré des propriétaires-actionnaires aux managers exécutifs, ces derniers sont susceptibles d'agir dans leur propre intérêt plutôt que dans celui des actionnaires. L'argument le plus souvent avancé est que ces managers sont 
davantage intéressés par le chiffre d'affaires de l'entreprise, sa taille et le nombre des emplois, qui sont autant de signes de pouvoir, que par la profitabilité des investissements. De là la revendication d'un mode de gouvernance qui donne toute latitude aux actionnaires dont on pense qu'ils sont seuls incités à maximiser le surplus, seuls aussi à pouvoir imposer les restruturations propres à rendre possible créativité et innovation. Or, mettre ainsi l'accent sur le contrôle fait oublier que l'objet de la délégation d'autorité aux managers exécutifs par des actionnaires dispersés, est de garantir l'engagement du capital auprès des autres acteurs de l'entreprise que sont les fournisseurs, les clients, les salariés, mais aussi les créanciers.

Aussi convient-il de distinguer les actionnaires de 'l'extérieur' des actionnaires 'de l'intérieur', les premiers ne s'intéressant qu'au dividende à percevoir et au cours de l'action, les seconds s'intéressant à l'avenir de la société et aux dividendes qu'ils espèrent percevoir dans l'avenir, les premiers voulant encaisser un dividende aussi élevé que possible le plus vite possible, alors qu'il est dans l'intérêt des seconds que les profits soient retenus dans l'entreprise. Ainsi, le degré d'engagement dépend-il de la structure de l'actionnariat. Un actionnariat individuel dispersé ou la concentration de la propriété du capital entre les mains d'une famille sont une garantie de stabilité de l'engagement. La détention de volumes d'actions élevés par des fonds d'investissement activistes peut, au contraire, constituer un facteur de fragilité de l'entreprise et de volatilité des cours rendant instable la capacité d'engagement. De là, la nécessité de faire valoir l'importance d'un actionnariat stable dans le but de garantir la capacité d'innovation (Mayer 2013, Bolton et Samama 2013).

La capacité d'innover (de créer de la valeur) procède d'investissements réels garants de l'apprentissage des conditions techniques et des conditions de marché, mais aussi de la cohérence des choix effectués. Les moyens financiers qui sont engagés pour couvrir les dépenses doivent être disponibles dans les montants et aux moments requis. C'est le sens qu'il convient de donner à la notion de liquidité, à savoir la détention d'actifs financiers de réserve qui relève de la capacité maintenue d'emprunter et d'émettre de nouveaux titres sur les marchés. C'est, en outre, cette capacité qui valide les engagements pris et font la valeur de l'entreprise.

La neutralité de la structure de financement énoncée par Modigliani et Miller (1958, 1988) est remise en cause, non pas en raison de l'existence de frictions que sont les taxes 
ou le défaut d'information des investisseurs sur les résultats actuels ou futurs de l'entreprise, mais parce que l'intérêt de l'entreprise n'est pas réductible à celui de ses actionnaires. De fait, la valeur de l'entreprise dépend de la structure de son financement dans la mesure où celle-ci affecte l'engagement des détenteurs de capitaux et, par suite, celui des autres parties prenantes à commencer par les salariés.

\section{Les ressources humaines entre flexibilité et apprentissage}

En même temps que la notion de créativité est mise en avant plutôt que celle d'innovation, la ressource humaine redevient, dans l'esprit des décideurs, une ressource individuelle plutôt que collective. Les mesures de transition vers l'emploi, censées assurer une sécurité professionnelle individuelle, l'emportent sur les garanties de maintien dans l'emploi. La fréquence des changements d'emploi et donc des passages par le marché externe augmente en même temps que baisse la durée moyenne des emplois. Tout cela n'a évidemment de justification que si la capacité d'innovation des entreprises s'en trouve accrue. Or il est possible sinon vraisemblable que ce soit l'inverse qui se produise.

Les effets pervers de la flexibilité des relations de travail

Le principal résultat de la flexibilité des marchés de travail est, aujourd'hui, une polarisation durable entre emplois très qualifiés, fortement rémunérés, et emplois non qualifiés, faiblement rémunérés, avec à la clé une baisse du salaire médian. Les ressources libérées, loin d'être dirigées vers les activités de haute technologie mieux rémunérées, sont contraintes d'aller vers des activités où les emplois offerts sont peu ou faiblement qualifiés, parfois à temps partiel et le plus souvent précaires. Ne serait-ce que parce que les travailleurs livrés à eux-mêmes ne disposent pas des moyens financiers suffisants, ni des connaissances leur permettant d'accéder aux qualifications supérieures demandées. La multiplicité des emplois occupés au cours de la vie professionnelle pourrait, alors, relever de cette précarité plutôt que de refléter la multiplicité des métiers exercés et des qualifications détenues au cours d'une vie professionnelle. Auquel cas, les forts taux d'entrée et de sortie seraient révélateurs de la précarité des emplois plus que de l'intensité et de la rapidité des innovations, la diminution du taux de chômage irait de pair avec l'augmentation du taux d'emploi précaire et du taux de pauvreté.

De cette polarisation témoignent les études empiriques de la situation de l'emploi réalisées depuis deux décennies notamment aux États-Unis (Katz et Krueger 2016). Un très grand nombre des millions d'emplois créés ont été des emplois d'intérim, des emplois 
ponctuels, des emplois en contrats courts, des emplois de contractants indépendants. Le plus souvent les travailleurs pauvres, peu ou pas qualifiés, naviguent entre chômage et emplois précaires du fait de leurs faibles chances de bénéficier d'une formation pendant leur période d'emploi.

Cette transformation de la nature des emplois et la chute des salaires qui l'accompagne affectent la qualité des ressources humaines et, par suite la croissance potentielle. Ce ne sont pas les rigidités du marché du travail qui orientent les choix d'investissement et les choix technologiques que ces investissements portent dans un sens défavorable à la productivité et à la croissance, mais le développement du dualisme sur les marchés du travail. La polarisation des emplois et des salaires va de pair avec celle des types d'entrepreneuriat.

Encourager une flexibilité de réaction, autoriser institutionnellement de larges fluctuations de l'emploi, c'est, paraît-il, encourager les entreprises à choisir des investissements plus risqués puisqu'elles n'auront pas à supporter la totalité de ces risques dont une partie sera supportée par les travailleurs à rebours de la thèse qui veut seuls les actionnaires seraient exposés au risque. Mais c'est surtout inciter ces mêmes entreprises à ne pas investir beaucoup en capital humain, dès lors qu'elles anticiperaient pouvoir perdre le bénéfice de cet investissement avec le départ, volontaire ou involontaire, de leurs salariés. D’une certaine façon, les mauvaises entreprises pourraient chasser les bonnes. La créativité s'exerce avec la seule ambition de réaliser des gains à très court terme.

\section{Éloge de la rigidité des relations de travail}

Cette approche ignore que la relation de travail s'est transformée en même temps que se développait l'entreprise moderne. Est ainsi apparu le contrat de travail dont la caractéristique, souvent oubliée dans la littérature de théorie économique, est qu'il n'est pas une stricte relation de marché comme l'est le contrat de louage. Il constitue une relation d'autorité avant d'être une relation marchande (Simon 1951). En fait, il suppose une certaine continuité de la relation entre employeur et employé. Cette continuité explique qu'il existe un coût à la rupture de cette relation, pour l'employé bien sûr, mais aussi pour l'employeur dès lors qu'il y a, au détriment de l'un comme de l'autre, perte du capital humain accumulé et de la capacité d'apprentissage que ce dernier recèle. En d'autres termes, le contrat de travail s'inscrit dans la perspective d'un enrichissement 
progressif des compétences répondant à la nécessité, pour l'entreprise, de renouveler en permanence ses métiers (Segrestin et Hatchuel 2012).

La flexibilité propre au contrat de travail tient alors à ce que les tâches fixées par l'employeur peuvent changer et changent généralement pour répondre à de nouveaux besoins des entreprises. Elle constitue une forme de liquidité visant, à l'instar des actifs financiers de réserve, à préserver la gamme, la plus large possible, des choix futurs (Simon 1951). Elle repose sur la conviction que l'innovation, entendue comme une activité récurrente, est une affaire d'apprentissage collectif au cœur de laquelle se trouve l'entreprise.

La durabilité et la flexibilité de la relation d'emploi requièrent une confiance réciproque entre l'employeur et l'employé, laquelle repose sur l'équité du contrat de salaire (Hicks 1974 p. 64). L'équité n'est pas une notion simple, mais, à tout le moins, elle suppose une relative rigidité du salaire, qui est affaire de continuité, non d'illusion monétaire (ibid. p. 66).

La durabilité de la relation d'emploi (du contrat de travail) est, en outre, conditionnée par la durabilité des contrats de financement. Ce n'est que si l'entrepreneur a la garantie de disposer des moyens de financement nécessaires au moment où ils sont requis qu'il peut garantir aux travailleurs la durée de leur emploi.

On l'aura compris, la flexibilité requise n'est pas celle dont on parle habituellement. Elle n'est pas assimilable à une réactivité immédiate des taux de salaire aux signaux du marché génératrice de mobilité que rendraient possible les facilités d'embauche et de licenciement. Elle devient une capacité d'initiative permettant à l'entreprise d'évoluer, de créer de nouvelles options productives et de construire son propre environnement en créant de nouvelles qualifications et compétences (Amendola et Bruno 1990).

L'accent est, alors, mis sur le potentiel que recèlent, à terme, les ressources humaines plutôt que sur leur performance immédiate. Ce qui importe, ce sont moins les tâches assignées à un moment déterminé aux travailleurs que la possibilité de leur redéploiement en vue de remplir de nouvelles tâches. L'externalisation de certaines d'entre elles a pour objectif, non de baisser les coûts, mais de libérer des ressources internes pour les affecter à de nouvelles tâches plus productives ou plus prometteuses. Ces processus d'apprentissage et de redéploiement prennent du temps et impliquent des 
hausses temporaires de coût, mobilisent des ressources sans que leur usage soit systématiquement et immédiatement optimisé.

Certes, dans le domaine des nouvelles technologies, de facto, les entreprises recourent principalement au marché externe du travail avec comme conséquence que la durée dans l'emploi est relativement faible. Ces entreprises procèdent ainsi parce qu'elles sont à la recherche des expériences acquises par leurs salariés chez leurs concurrents. Elles proposent, alors, des salaires sans cesse plus élevés à des salariés qui exercent leur pouvoir de marché et restent durablement employés bien que ce soit dans différentes entreprises. Elles peuvent le faire parce ce qu'elles se trouvent en position de concurrence monopolistique. Aussi est-ce d'une mobilité très particulière du travail dont il s'agit, qu'il faut distinguer de celle rendue nécessaire par les destructions d'emplois du fait de changements de technologies ou de préférences des clients induisant des changements de qualification. Tout aussi particulière est la mobilité induite par l'existence de communautés de pratiques ou de savoirs, associant des salariés pouvant travailler dans différentes entreprises, dont l'objet est la création de connaissances. Ces communautés transcendent la distinction entre marchés internes et externes du travail en même temps qu'elles stabilisent les engagements individuels, bien loin de la flexibilité dans son acception commune d'optimisation immédiate des situations individuelles. Elles sont le reflet de la volonté de maîtriser les interactions sociales, non pas à un moment donné du temps, mais tout au long d'un processus d'innovation jamais parvenu à son terme.

\section{Conclusion}

La créativité s'avère être un concept délicat à manier d'un point de vue d'analyse économique. Comme l'entrepreneuriat, elle peut s'exercer dans le sens de la création comme du détournement de valeur. C'est sans doute la raison pour laquelle le concept d'innovation lui a été le plus souvent préféré en économie. Avec, cependant, un défaut qui est, précisément, d'ignorer que des activités entrepreneuriales et donc la créativité relèvent, possiblement, de la captation de rente au détriment de l'investissement productif. Avec le défaut conjoint de voir dans l'innovation un acte unique plutôt que de considérer la séquence des innovations successives. Ce faisant, le pouvoir de marché est présenté, systématiquement, comme un obstacle à la créativité et à l'innovation, et le marché pleinement concurrentiel loué y compris en imaginant que les nouvelles 
technologies se prêtent à son existence. Alors que ce qui est en jeu reste l'usage qui est fait des ressources disponibles et donc du pouvoir de marché comme de l'esprit créatif.

La réalité de la créativité réside dans le choix opéré entre un entrepreneuriat à la recherche de rentes et un entrepreneuriat pourvoyeur d'innovations productives, entre une finance impatiente et une finance patiente, entre flexibilité et rigidité sur les marchés de travail. Ce choix est d'ordre organisationnel et institutionnel. Deux types de société de marché, toutes deux créatives mais pour des buts et avec des résultats différents, sont ainsi mises en balance, qui se distinguent, certes par le rôle reconnu à l'État, mais aussi par le mode d'organisation des entreprises, de la finance et du travail (Amendola et Gaffard 2018, 2019, Gaffard, Amendola et Saraceno 2020). L’une est le néo-libéralisme qui a pris place à partir des années 1980, l'autre le libéralisme social (ou socialisme libéral) tel qu'il a pu se développer après la grande Dépression et le second conflit mondial. L'une fait usage des connaissances scientifiques et technologiques existantes dans le but de capter rapidement des rentes au bénéfice d'un petit nombre, et se trouve ainsi bien loin d'une société de petits propriétaires. L'autre est créatrice de connaissances et de technologies procédant d'une coopération entre les parties prenantes constitutive de l'entreprise et complément nécessaire de la concurrence pour le bénéfice du plus grand nombre.

Le débat ainsi engagé sur la nature de la créativité s'inscrit dans la réflexion de philosophie sociale qui parcourt la théorie économique depuis les origines. Aristote dans le Politique ne distinguait-il pas entre la recherche de l'argent pour la satisfaction des besoins et la recherche de l'argent pour lui-même, entre la bonne et la mauvaise chrématistique? Keynes dans la Théorie générale comme dans le Traité sur la monnaie, s'inspirant explicitement de la pensée mercantiliste, ne se souciait-il pas des effets pervers d'une propension à investir trop systématiquement inférieure à la propension à épargner alimentant alors une circulation financière au détriment de la circulation industrielle ? Ce qui interroge au plan de la société dans son ensemble se décline aussi au niveau des entreprises et des marchés. 


\section{Références}

Amendola M. and S. Bruno (1990): 'The Behaviour of the Innovative Firm. Relations to the Environment', Research Policy 19: 419-433.

Amendola M. and J-L Gaffard (2018): Le désordre et la raison. Une économie politique de la mondialisation, Londres : ISTE.

Amendola M. and J-L Gaffard (2019): Disorder and Public Concern about Globalization, London: ISTE and Wiley.

Arrow K.J. (1974): The Limits of Organization, New York: Norton \& Cie.

Baumol W.J. (2002): The Free-Market Innovation Machine, Princeton, Princeton University Press.

Baldwin C.Y. et K. B. Clark (2001) : 'Modularity After Crash', Working Paper, Harvard Business School.

Bolton, P. and F. Samama (2013): 'L-Shares: Rewarding Long-Term Investors', ECGI Finance Working Paper No. 342/2013.

Gaffard J-L, Amendola M. et F. Saraceno (2020) : Le temps retrouvé. Instabilité et résilience des économies de marché, Paris, Odile Jacob.

Georgescu-Roegen N. (1971) : The Entropy Law and the Economic Process, Cambridge Mass., Harvard University Press,.

Granovetter M. (1985): « Economic Action and Social Structure: the Problem of Embeddedness», American Journal of Sociology 91 (3) : 481-510.

Hayek F.A. (1948): Individualism and Economic Order, Chicago, The University of Chicago Press.

Hicks J.R. (1974): The Crisis in Keynesian Economics, Oxford: Basil Blackwell.

Howitt P., « Macroeconomics with Intelligent Autonomous Agents », in R. Farmer (éd.), Macroeconomics in the Small and the Large, Essays in Honor of Axel Leijonhufvud, Cheltenham, Edward Elgar, 2008.

Katz L.F. and A.B. Krueger (2016): 'The Rise and Nature of Alternative Work Arrangements in the United States, 1995-2015', Working Paper. 
Lazonick W. (2007): 'Varieties of Capitalism and Innovative Enterprise', Comparative Social Research 24 : 21-69

Lazonick W. (2009): 'The New Economy Business Model and the Crisis of U.S. Capitalism', Capitalism and Society 4 (2): 1-67.

Loasby B.J. (1991): Equilibrium and Evolution. An exploration of connecting principles in economics, Manchester, Manchester University Press.

Mayer C., (2013) : Firm Commitment, Oxford, Oxford University Press.

March J. (1962): 'The Business Firm as a Political Coalition', The Journal of Politics 24 (4): 662-678.

Mazzucato M. (2013): 'Financing Innovation: Creative Destruction vs. Destructive Creation', Industrial and Corporate Change 22 (4): 851-867

Mazzucato M. (2014): The Entrepreneurial State. Debunking Public vs Private Sector Myths, London, Anthem.

Metcalfe J.S. (2001): 'Institutions and Progress', Industrial and Corporate Change 10 (3): 561-586.

Modigliani F. and M. Miller (1958): 'The Cost of Capital, Corporation Finance and the Theory of Investment', American Economic Review 48: 261-297.

Modigliani F. and M. Miller (1988): 'The Modigliani -Miller Propositions After Thirty Years', Journal of Economic Perspectives 2 (4): 99-120.

Penrose E. (1959): The Theory of the Growth of the Firm, Oxford: Oxford University Press. $3^{\text {rd }}$ edition 1995.

Phelps E. (2013): Mass Flourishing, Princeton, Princeton University Press; traduction française, La prospérité de masse, Paris, Odile Jacob, 2017.

Richardson G. B. (1960) : Information and Investment, A study in the working of the competitive economy, Oxford : Oxford University Press. Reed.1990.

Schumpeter J.A. (1934): Theory of Economic Development, Cambridge Mass.: Harvard University Press. Traduction française, Théorie du développement économique, Paris: Payot. 
Schumpeter J.A. (1941): Capitalism, Socialism, and Democracy, New York, Harper \& Row. Traduction française, Capitalisme, Socialisme et Démocratie, Paris: Payot.

Segrestin B. et A. Hatchuel (2012) : Refonder l'entreprise, Paris : Le Seuil.

Shackle G.L.S. (1949): Expectations in Economics, Cambridge: Cambridge University Press.

Shackle G.L.S. (1955): Uncertainty in Economics and Other Reflections, Cambridge, Cambridge University Press.

Simon H.A. (1951) : 'A Formal Theory of the Employment Relationship', Econometrica 19 (3) : 293-305.

Skidelsky R., Keynes : the return of the master, New York, Public Affairs, 2009.

Smith A. (1776) : An Inquiry in the Nature and Causes of the Wealth of Nations, Reed. (1976) R.H. Campbell and A.S. Skinner (eds), Oxford : Clarendon Press. 


\section{ABOUT OFCE}

The Paris-based Observatoire français des conjonctures économiques (OFCE), or French Economic Observatory is an independent and publicly-funded centre whose activities focus on economic research, forecasting and the evaluation of public policy.

Its 1981 founding charter established it as part of the French Fondation nationale des sciences politiques (Sciences Po), and gave it the mission is to "ensure that the fruits of scientific rigour and academic independence serve the public debate about the economy". The OFCE fulfils this mission by conducting theoretical and empirical studies, taking part in international scientific networks, and assuring a regular presence in the media through close cooperation with the French and European public authorities. The work of the OFCE covers most fields of economic analysis, from macroeconomics, growth, social welfare programmes, taxation and employment policy to sustainable development, competition, innovation and regulatory affairs.

\section{ABOUT SCIENCES PO}

Sciences $\mathrm{Po}$ is an institution of higher education and research in the humanities and social sciences. Its work in law, economics, history, political science and sociology is pursued through ten research units and several crosscutting programmes.

Its research community includes over two hundred twenty members and three hundred fifty PhD candidates. Recognized internationally, their work covers a wide range of topics including education, democracies, urban development, globalization and public health.

One of Sciences Po's key objectives is to make a significant contribution to methodological, epistemological and theoretical advances in the humanities and social sciences. Sciences Po's mission is also to share the results of its research with the international research community, students, and more broadly, society as a whole. 